\title{
$\mathbf{J}|\mathbf{A}| \mathbf{C} \mid \mathbf{S}$ \\ COMMUNICATIONS
}

Published on Web 03/13/2002

\section{Selective Binding of Mannose-Encapsulated Gold Nanoparticles to Type 1 Pili in Escherichia coli}

\author{
Chun-Cheng Lin, ${ }^{*}+$ Yi-Chun Yeh, ${ }^{\ddagger}$ Chan-Yi Yang, ${ }^{\ddagger}$ Chan-Long Chen, ${ }^{\ddagger}$ Gee-Fong Chen, ${ }^{\ddagger}$ \\ Chia-Chun Chen, ${ }^{*}, \neq$ and $\mathrm{Yi}-\mathrm{Ch} u n \mathrm{Wu}^{*}, \S$ \\ Institute of Chemistry, Academia Sinica, Nankang, Taipei 115, Taiwan, Department of Chemistry, \\ National Taiwan Normal University, Taipei 116, Taiwan, and Department of Zoology, \\ National Taiwan University, Taipei 106, Taiwan
}

Received January 17, 2002

Metal and semiconductor nanoparticles coupled with biomolecules have attracted great interests recently because the resulting materials may bring new applications in biological systems. ${ }^{1}$ In protein-based recognition, many current diagnostic kits have been long developed on the basis of the interaction between antibody conjugated gold nanoparticles and their antigens. ${ }^{2}$ Of late, gold nanoparticles attached with DNA have been applied for the detection of target sequence through complementary hybridization. ${ }^{3}$ Also, functional organic ligands have been encapsulated on gold nanoparticles for the fabrication of new chemical probes. ${ }^{4}$ Besides gold nanoparticles, semiconductor nanoparticle bioconjugates as selective fluorescent biological labels have shown great potential in biological studies and medical applications. ${ }^{5}$ However, the biomolecules on functionalized nanoparticles in recent studies are mostly DNA or protein, and very few examples ${ }^{6}$ have been reported on carbohydrates. Moreover, the application of carbohydrate-conjugated nanoparticles in biological assays has not been explored.

The technical advantages of applying gold nanoparticles in biological systems have been well recognized. ${ }^{2}$ The covalent binding between gold nanoparticles and biomolecules can be easily achieved by self-assembled thiolated molecules onto the nanoparticle surface. ${ }^{7}$ In addition, gold nanoparticles exhibit an intense color in visible region for spectroscopic detection and also great contrast for electron microscopic imaging. ${ }^{2}$ Moreover, a single nanoparticle with large surface volume ratio is ready for the covalent attachment of multiple ligands, ${ }^{5}$ which provides a great possibility for the enhancement of some biomolecule interactions. In particular, carbohydrate-protein interaction is generally identified with very low affinity between each other. However, in nature, the low affinity can be compensated by presentation of multiple ligands to individual receptors. The polyvalent interactions between multi-ligands and their receptors can be collectively much stronger than corresponding monovalent interactions. ${ }^{8}$ Recently, multiple carbohydrate ligands have been assembled on linear polymers, ${ }^{9}$ two-dimensional gold surface ${ }^{10}$ and liposomes ${ }^{11}$ to enhance carbohydrate-protein interactions. Herein, we report the synthesis and characterization of mannose-encapsulated gold nanoparticles (m-AuNP). m-AuNP binds specifically to FimH adhesin of bacterial type 1 pili as observed by transmission electron microscopy (TEM) and shows stronger interaction with FimH than free mannose does in the competition assay. This work demonstrates that carbohydrate-attached gold nanoparticles can be used as efficient labeling probe and multiligand carrier in a biological system.

* To whom correspondence should be addressed. E-mail: t42005@cc.ntnu.edu.tw.

$*$ To whom correspondence should be addres
$\dagger$ Institute of Chemistry, Academia Sinica.

$¥$ Department of Chemistry, National Taiwan Normal University.

$\S$ Department of Zoology, National Taiwan University.
Scheme 1. Synthesis of m-AuNP $1^{a}$

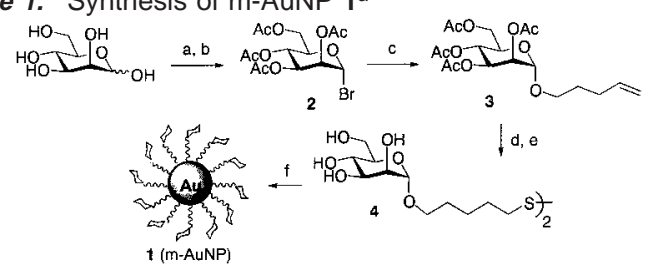

${ }^{a}$ Keys: (a) $\mathrm{Ac}_{2} \mathrm{O}$, pyr. DMAP, $90 \%$. (b) $\mathrm{HBr} / \mathrm{HOAc}, 80 \%$. (c) 4-pentenyl alcohol, $\mathrm{Hg}(\mathrm{CN})_{2}, 88 \%$ (d) $\mathrm{HSAc}$, AIBN, dioxane, $80 \%$. (e) $\mathrm{NaOMe}$ (cat.), $\mathrm{MeOH}, 97 \%$. (f) $\mathrm{HAuCl}_{4}, \mathrm{NaBH}_{4}$.

As shown in Scheme 1, compound $\mathbf{3}$ was synthesized by glycosylation of $\mathbf{2}$ with alcohol and then hydrolyzed to obtain thiomannosyl dimer $4 .^{12}$ The m-AuNP $\mathbf{1}$ was prepared by treating $\mathrm{HAuCl}_{4}$ with 4 in the presence of $\mathrm{NaBH}_{4}{ }^{13}$ The spherical m-AuNP with an average diameter of $6 \pm 1 \mathrm{~nm}$ were observed by TEM, and no aggregation was found in the images. Both UV-visible spectra of gold nanoparticles before and after coupling with mannose showed clear plasmon bands of $\lambda \approx 520 \mathrm{~nm} .{ }^{14}$ No red-shift or intensity decrease of the band ${ }^{15}$ after modification also indicated that no aggregation occurred in aqueous media. The X-ray photoelectron spectrum (XPS) of m-AuNP solid was different from that of unbound thiols $(\mathrm{S}-\mathrm{H}) .{ }^{16}$ Particularly, a large binding energy difference was observed for $\mathrm{S} 2 \mathrm{p}_{3 / 2}(\sim 1.8 \mathrm{eV})$. This result indicates that a thiolate $(\mathrm{S}-\mathrm{Au})$ is indeed present in $\mathrm{m}-\mathrm{AuNP}$. In addition, the formation of m-AuNP was also confirmed by the transmission IR and NMR spectra. A single m-AuNP consists of approximately 200 attached mannoses, as estimated from the average nanoparticle diameter and the result of elemental analysis. ${ }^{16}$

The stability of m-AuNP in various media was further examined to assess the possibility of its application in a biological system. Previous reports have shown that gold nanoparticles stabilized by surfactants or polymers were not effective in preventing aggregation of the nanoparticles particularly under high concentrations of salt medium. ${ }^{17}$ In contrast, m-AuNP was found to be very stable in deionized water and phosphate buffer solution (PBS), and its stability was independent of high ion strength and $\mathrm{pH}$ values in the range from 1.5 to 12 of solutions, as suggested by the absorption spectra. Moreover, $\mathrm{m}-\mathrm{AuNP}$ is easily redissolved in aqueous media without aggregation. These properties have made application of $\mathrm{m}$-AuNP in biological systems feasible.

m-AuNP was then tested for its ability to bind mannose-specific adhesin FimH of type 1 pili in Escherichia coli. Type 1 pili are filamentous proteinaceous appendages that extend from the surface of many gram-negative organisms and are composed of FimA, FimF, FimG, and FimH proteins. ${ }^{18}$ FimA accounts for more than $98 \%$ of the pilus protein, and FimH is uniquely responsible for the 

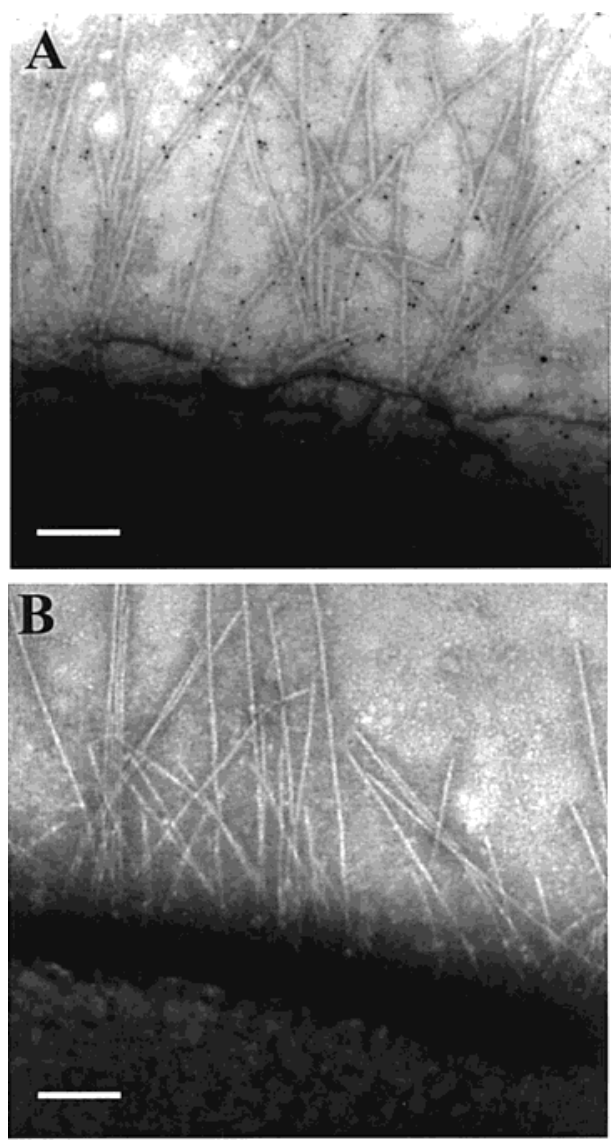

Figure 1. Typical TEM images of sectioned areas of (A) pili of the $E$. coli ORN178 strain bound with m-AuNP, (B) the E. coli ORN208 strain deficient of the $f i m H$ gene without $\mathrm{m}$-AuNP binding. The experiments were performed in LB at room temperature. Scale bar $=100 \mathrm{~nm}$.

binding to D-mannose. ${ }^{19}$ Two E. coli strains ORN178 and ORN208 were used in experiments to confirm the specific binding of m-AuNP to FimH. The ORN178 strain expresses wild-type type 1 pili, whereas the ORN208 strain is deficient of the fimH gene and expresses abnormal type 1 pili that fail to mediate D-mannosespecific binding. ${ }^{19 a}$ Two bacterial strains were incubated with $\mathrm{m}$-AuNP separately in buffers (PBS, $\mathrm{LB}$, and $\mathrm{H}_{2} \mathrm{O}$ ) and at temperatures $\left(4,25\right.$, and $\left.37^{\circ} \mathrm{C}\right)$, and the binding of $\mathrm{m}$-AuNP to bacterial pili in each condition was examined by TEM. The TEM results showed that m-AuNP selectively bound the pili of the ORN178 strain but not those of the ORN208 strain (Figure 1), demonstrating specific binding of $\mathrm{m}$-AuNP to FimH. The selective binding of the ORN 178 was observed in all buffers and temperatures tested. However, the best result was obtained at $25^{\circ} \mathrm{C}$. The nanoparticles were localized at the lateral ends and distributed at intervals along the shaft of the pili (on the average of 100-150 nm interval) in ORN178 strain, consistent with the localization pattern of FimH protein along type 1 pili viewed by immunoelectron microscopy. ${ }^{19 b}$

Further experiments were performed to test the binding ability of m-AuNP to FimH with respect to free mannose in solution. Specifically, methyl $\alpha$-D-mannopyranoside was used as competitor of $\mathrm{m}$-AuNP for FimH in binding experiments. Mannose at various concentrations and m-AuNP were co-incubated with ORN178 E. coli to reach an equilibrium, and the binding of $\mathrm{m}$-AuNP to bacterial pili was examined using TEM. Free mannose at concentrations up to twenty times of m-AuNP concentration had no or little effect on the binding of $\mathrm{m}$-AuNP to bacterial pili. Mannose concentrations required for competing out $\sim 10$ and $\sim 90 \%$ of $\mathrm{m}$-AuNP binding to bacterial pili were approximately 100 and 2000 times of m-AuNP concentration, respectively. These results suggest that m-AuNP binds FimH better than free mannose does. Currently, several binding assays such as BIACore SPR are performed to establish a binding model ${ }^{8-11}$ between $\mathrm{m}$-AuNP and FimH in detail.

The strong and selective binding of $\mathrm{m}$-AuNP to bacterial type 1 pili presents a novel method of labeling specific protein on the cell surface using carbohydrate conjugated nanoparticles. Moreover, in comparison with the conventional sandwich immunoassay, the biomolecule conjugated nanoparticles can provide a relatively easy and direct method to visualize the target receptors on the cell surface under an electron microscope. Other potential applications of carbohydrate-encapsulated nanoparticles, for example, to block the protein-carbohydrate adhesion may provide new insights for the design of pharmaceutical active agents.

Acknowledgment. We acknowledge financial support from the NSC, Academia Sinica, NTNU (ORD91-1), NINT project (C.C.C.), and Ministry of Education (89-B-FA-01-1-4) (Y.C.W.). We thank Professor P. E. Orndorff for E. coli strains and Professor S.-H. Chien and Y.-S. Yang for XPS measurements.

Supporting Information Available: Detailed experimental procedures, stability measurements, competition assays, and data analyses for UV-vis, TEM, XPS, IR, and NMR spectroscopy (PDF). This material is available free of charge via the Internet at http://pubs.acs.org.

\section{References}

(1) For a recent review, see: Niemeyer, C. M. Angew. Chem., Int. Ed. 2001 40, 4128-4258

(2) (a) Hayat, M. A. Colloidal Gold: Principles, Methods and Applications; Academic Press: New York, 1989. (b) Kreuter, J. In Microcapsules and Nanoparticles in Medicine and Pharmacy; Donbrow, M., Ed.; CRC: Boca Raton, 1992.

(3) (a) Alivisatos, A. P.; Peng, X.; Wilson, T. E.; Johnsson, K. P.; Loweth C. J.; Bruchez, M. P., Jr.; Schultz, P. G. Nature 1996, 382, 609-611. (b) Mirkin, C. A.; Letsinger, R. L.; Mucic, R. C.; Storhoff, J. J. Nature 1996 382, 607-609. (c) Taton, T. A.; Lu, G.; Mirkin, C. A. J. Am. Chem. Soc. 2001, 123, 5164-5165. (d) Taton, T. A.; Mirkin, C. A.; Letsinger, R. L. Science 2000, 289, 1757-1760.

(4) (a) Boal, A. K.; Ilhan, F.; DeRouchey, J. E.; Thurn-Albrecht, T.; Russell, T. P.; Rotello, V. M. Nature 2000, 404, 746-748. (b) Boal, A. K.; Rotello, V. M. J. Am. Chem. Soc. 2000, 122, 734-735.

(5) (a) Bruchez, M. P., Jr.; Moronne, M.; Gin, P.; Weiss, S.; Alivisatos, A. P. Science 1998, 281, 2013-2016. (b) Chan, W. C. W.; Nie, S. Science 1998, 281, 2016-2018.

(6) (a) de la Fuente, J. M.; Barrientos, A. G.; Rojas, T. C.; Rojo, J.; Cañada, J.; Fernández, A.; Penadés, S. Angew. Chem., Int. Ed. 2001, 40, 22582261. (b) Otsuka, H.; Akiyama, Y.; Nagasaki, Y.; Kataoka, K. J. Am. Chem. Soc. 2001, 123, 8226-8230.

(7) Ulman, A. Chem. Rev. 1996, 96, 1533-1554.

(8) Mammen, M.; Choi, S.-K.; Whitesides, G. M. Angew. Chem., Int. Ed. 1998, 37, 2755-2794.

(9) Gestwicki, J. E.; Kiessling, L. L. Nature 2002, 415, 81-84.

(10) (a) Liang, M. N.; Smith, S. P.; Metallo, S. J.; Choi, I. S.; Prentiss, M.; Whitesides, G. M. Proc. Natl. Acad. Sci. U.S.A. 2000, 97, 13092-13096. (b) Mann, D. A.; Kanai, M.; Maly, D. J.; Kiessling, L. L. J. Am. Chem. Soc. 1998, 120, 10575-10582.

(11) Kingery-Wood, J. E.; Williams, K. W.; Sigal, G. B.; Whitesides, G. M J. Am. Chem. Soc. 1992, 114, 7303-7305.

(12) (a) Kartha, K. P. R.; Jennings, H. J. J. Carbohydr. Chem. 1990, 9, 777781. (b) Lee, R. T.; Lee, Y. C. Carbohydr. Res. 1995, 271, 131-136. (c) Buskas, T.; Söderberg, E.; Konradsson, P.; Fraser-Reid, B. J. Org. Chem 2000, 65, 958-963.

(13) Brust, M.; Walker, M.; Betthell, D.; Schiffrin, D. J.; Whyman, R. J. Chem Soc., Chem. Commun. 1994, 801-802.

(14) Keating, C. D.; Kovaleski, K. M.; Natan, M. J. J. Phys. Chem. B 1998 , 102, 9404-9413, and reference therein.

(15) Storhoff, J. J.; Lazarides, A. A.; Mucic, R. C.; Mirkin, C. A.; Letsinger R. L.; Schatz, G. C. J. Am. Chem. Soc. 2000, 122, 4640-4650.

(16) Heister, K.; Zharnikov, M.; Grunze, M.; Johansson, L. S. O. J. Phys. Chem. B 2001, 105, 4058-4061.

(17) Sato, T.; Ruth, R. Stabilization of Colloidal Dispersions by Polymer Adsorption; Surfactant Science Series, No.9; Marcel Dekker: New York 1980; pp 65-119.

(18) Sato, G. E.; Hultgren, S. J. J. Bacteriol. 1999, 181, 1059-1071.

(19) (a) Harris, S. L.; Spears, P. A.; Havell, E. A.; Hamrick, T. S.; Horton, J. R.; Orndorff P. E. J. Bacteriol. 2001, 183, 4099-4102. (b) Krogfelt, K. A.; Bergmans, H.; Klemm, P. Infect. Immun. 1990, 58, 1995-1998.

JA0200903 